\title{
A novel technique for biliary biopsy using the sheath of a plastic stent and a non-return valve
}

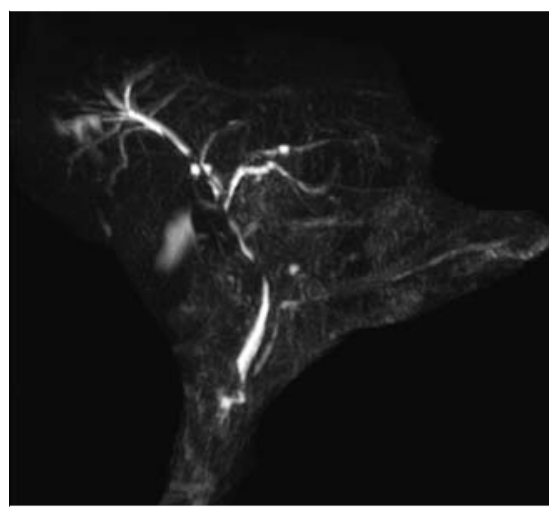

- Fig. 1 Magnetic resonance cholangiopancreatography (MRCP) showing multiple biliary stenoses.

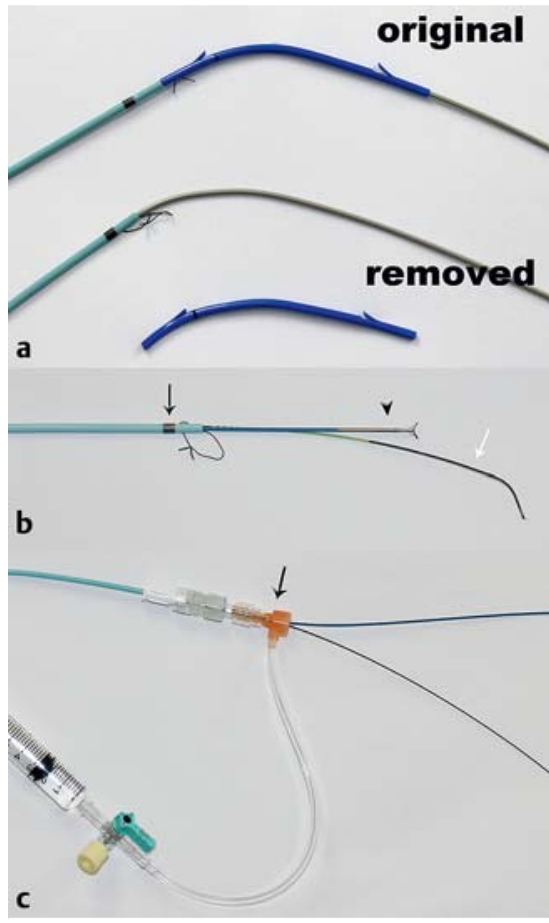

- Fig. 2 The equipment used to perform the biliary biopsy. a The system of a 7-Fr biliary plastic stent with the stent removed. $\mathbf{b}$ The tip of the outer sheath has a radiopaque marker (black arrow). The mini-forceps (arrowhead) and guidewire (white arrow) are passed into the sheath together. c The end of the outer sheath with a non-return valve (black arrow).
Endoscopic transpapillary biopsy has been widely performed for the diagnosis of biliary disease. Direct-vision biliary biopsy using peroral cholangioscopy has been reported to be very useful [1-4]. At centers where peroral cholangioscopy is unavailable, biliary biopsy can be performed under fluoroscopic guidance. It is however sometimes difficult to obtain adequate biopsy specimens owing to issues with guidance of the biopsy forceps. Hijioka et al. reported a useful technique for biliary biopsy using miniforceps with a biliary dilator [5]. Herein, we report a useful modification of their procedure.

A 70-year-old man with obstructive jaundice due to gallbladder cancer ( Fig.1) was referred to our hospital for right-sided hemihepatectomy. Biopsy specimens from the left biliary duct were needed to determine the extent of liver resection required. The system of a 7-Fr biliary plastic stent (Flexima; Boston Scientific, Marlborough, Massachusetts, USA) with the stent removed was used to perform the biopsy ( $\mathbf{F i g . 2}$ a).

Without a sphincterotomy having been performed, the system was inserted into the biliary duct over a guidewire. The guidewire allowed the system to be ad-

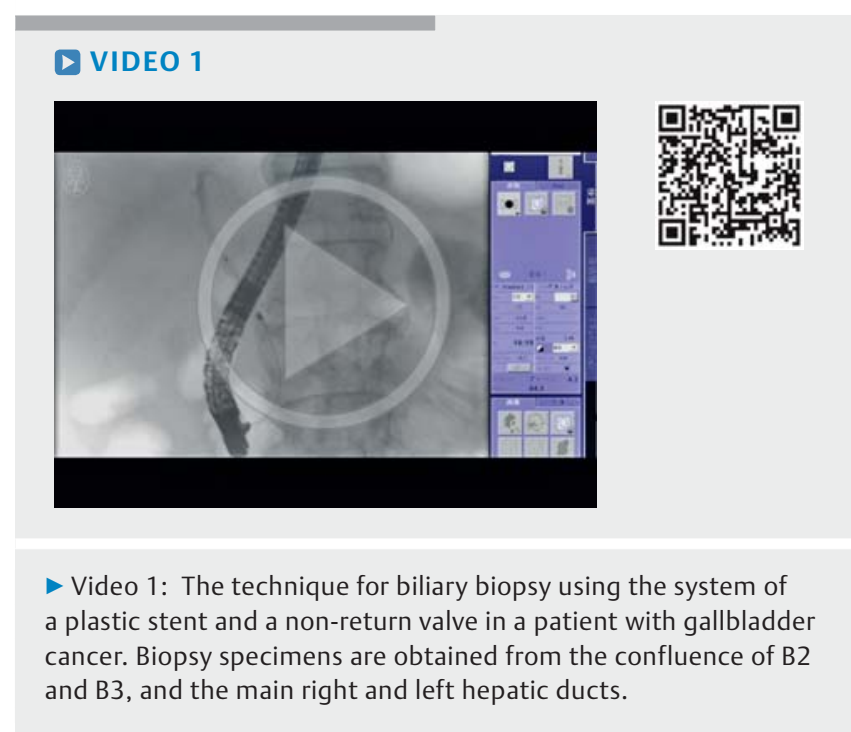

vanced to the biopsy site, and the inner sheath assisted passage through the stenotic area. At the biopsy site, the inner sheath was removed and a mini-forceps (SpyByte; Boston Scientific) was introduced into the outer sheath, which remained on the guidewire. The tip of the outer sheath had a radiopaque marker to allow identification of the exact exit point of the forceps fluoroscopically ( $\triangleright$ Fig.2b). Furthermore, attachment of a non-return valve (Terumo, Tokyo, Japan) to the end of the sheath ( $\mathbf{F i g . 2} \mathbf{c}$ ) allowed contrast medium to be injected into the biliary duct to recognize the exact biopsy site, without removal of the forceps, and a biopsy was successfully taken ( $\triangleright$ Fig. 3 ; $\triangleright$ Video 1 ). Finally, the plastic stent was re-mounted on the system and placed at the biliary stenosis. We believe that this technique would be useful at centers where peroral cholangioscopy is unavailable.

Endoscopy_UCTN_Code_TTT_1AR_2AD

\section{Competing interests}

None 


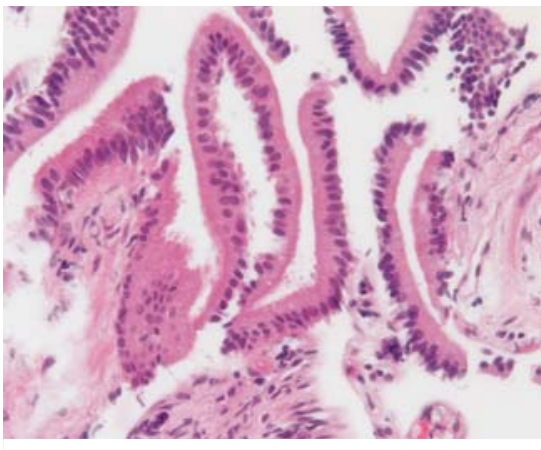

- Fig. 3 Photomicrograph of a biopsy specimen from the confluence of the main right and left hepatic ducts showing no malignant findings (hematoxylin and eosin [H\&E] staining; magnification $\times 40$ ).
The Authors

Shinichi Morita', Tsutomu Kanefuji ${ }^{1}$, Takahiro Hoshi', Masaaki Kobayashi', Takeshi Suda ${ }^{1}$, Takeshi Mizusawa²,

\section{Shuji Terai ${ }^{3}$}

1 Department of Gastroenterology and Hepatology, Uonuma Institute of Community Medicine Niigata University Hospital, Niigata, Japan

2 Department of Gastroenterology and Hepatology, Niigata Prefectural Tokamachi Hospital, Niigata, Japan

3 Department of Gastroenterology and Hepatology, Niigata University Hospital, Niigata, Japan

\section{Corresponding author}

\section{Shinichi Morita, MD}

Department of Gastroenterology and Hepatology, Uonuma Institute of Community Medicine Niigata University Hospital, 4132 Urasa, Minamiuonuma City, Niigata 949-7302, Japan,

Fax: +81-25-7772811,

m0riz0u@extra.ocn.ne.jp

\section{References}

[1] Kawakami H, Kuwatani M, Etoh K et al. Endoscopic retrograde cholangiography versus peroral cholangioscopy to evaluate intraepithelial tumor spread in biliary cancer. Endoscopy 2009; 41: 1-2

[2] Draganov PV, Chauhan S, Wagh MS et al. Diagnostic accuracy of conventional and cholangioscopy-guided sampling of indeterminate biliary lesions at the time of ERCP: a prospective, long-term follow-up study. Gastrointest Endosc 2012; 75: 347-353

[3] Nishikawa T, Tsuyuguchi T, Sakai Y et al. Comparison of the diagnostic accuracy of peroral video-cholangioscopic visual findings and cholangioscopy-guided forceps biopsy findings for indeterminate biliary lesions: a prospective study. Gastrointest Endosc 2013; 77: 219-226

[4] Osanai M, Itoi T, Igarashi Y et al. Peroral video cholangioscopy to evaluate indeterminate bile duct lesions and preoperative mucosal cancerous extension: a prospective multicenter study. Endoscopy 2013; 45: 635-642

[5] Hijioka S, Hara K, Mizuno N et al. A novel technique for endoscopic transpapillary "mapping biopsy specimens" of superficial intraductal spread of bile duct carcinoma (with videos). Gastrointest Endosc 2014; 79 : $1020-1025$

\section{Bibliography}

DOI http://dx.doi.org/10.1055/s-0042-118704

Endoscopy 2017; 49: E9-E10

(c) Georg Thieme Verlag KG

Stuttgart - New York

ISSN 0013-726X 